\title{
Long-lived higher excited state luminescence from new ruthenium(II)-allenylidene complexes
}

\author{
Joris van Slageren ${ }^{\mathrm{a}, *, 1}$, Rainer F. Winter ${ }^{\mathrm{b}, *}$, Axel Klein ${ }^{\mathrm{b}}$, Stephan Hartmann ${ }^{\mathrm{b}}$ \\ a Institute of Molecular Chemistry, Universiteit van Amsterdam, Nieuwe Achtergracht 166, NL-1018 WV Amsterdam, The Netherlands \\ ${ }^{\mathrm{b}}$ Institut für Anorganische Chemie der Universität Stuttgart, Pfaffenwaldring 55, D-70569 Stuttgart, Germany
}

\begin{abstract}
Resonance Raman spectra of heteroatom substituted ruthenium(II)-allenylidene complexes, obtained by irradiation into the second electronic absorption band, clearly prove the $\mathrm{d}(\mathrm{Ru}) \rightarrow \pi^{*}(\mathrm{CCC})$ MLCT character of the corresponding electronic transition. The complexes are not significantly luminescent at room temperature, but in solvent glasses at $77 \mathrm{~K}$, emission is observed. Only some of the complexes studied are luminescent upon irradiation into their lowest-energy absorption band. The striking finding of this study is that almost all complexes are luminescent on irradiation into their second absorption band. The emission was shown to originate from a higher lying ${ }^{3} \mathrm{MLCT}$ state, which shows that internal conversion to the lowest excited state is very inefficient in these complexes.
\end{abstract}

Keywords: Ruthenium(II); Allenylidene complexes; Photophysics; Luminescence; Resonance Raman

\section{Introduction}

Transition metal alkynyl complexes, in particular those of platinum(II), are often quite luminescent, which makes them good candidates in luminophore applications on their own or as emitting components in supramolecular systems [1,2]. Many of these systems contain a ligand with low-lying empty orbitals, usually an $\alpha$-diimine, in addition to the alkynyl ligands. The lowest-energy electronic transition and the corresponding relaxed excited states of those complexes are usually thought to have MLCT character. Recently, DFT calculations showed that in reality the low lying electronic transitions are more adequately described as ligand-to-ligand charge transfer ( $\mathrm{L}^{\prime} \mathrm{LCT}$ ), originating from the alkynyl ligands $[3,4]$. The shift of the $v(\mathrm{C} \equiv \mathrm{C})$

\footnotetext{
* Corresponding authors.
}

E-mail addresses: slageren@pil.physik.uni-stuttgart.de (J. van Slageren),winter@iac.uni-stuttgart.de (R.F. Winter).

1 Present address: Physikalisches Institut der Universität Stuttgart, Pfaffenwaldring 57, D-70550 Stuttgart, Germany; tel.: +49-711-6854193; fax: +49-711-685-4942 (J.V.S.) tel.: +49-711-685-4193; fax: +49-711-685-4165 (R.W.). frequency in the excited state with respect to the ground state shows that also in the relaxed excited state the alkynyl moiety plays a major role [5]. Other examples of luminescent transition metal-alkynyl systems include a series of alkynyl-rhenium(I) $\alpha$-diimine complexes [6], and mono- or dinuclear alkynyl-gold(I) complexes [7] or polymers [8]. Several luminescent transition metalalkynyl systems exist without such an acceptor ligand. Particularly, the $\left[\mathrm{Pt}(\mathrm{C} \equiv \mathrm{CR})_{2}(\mathrm{P})_{2}\right]$ complexes, where $(\mathrm{P})_{2}$ is one bidentate diphosphine or two simple phosphine ligands, have been studied in detail. Luminescence and resonance Raman studies proved the MLCT $\left(\mathrm{d}_{\pi}(\mathrm{Pt}) \rightarrow\right.$ $\left.\pi^{*}(\mathrm{C} \equiv \mathrm{C})\right)$ character of the lowest-energy electronic transitions and excited states [9].

Recently a series of ruthenium(II)-aminoallenylidene complexes trans $-\left[\mathrm{Cl}\left(\mathrm{L}_{2}\right)_{2} \mathrm{RuC}_{3}\left(\mathrm{NR}_{2}\right) \mathrm{R}^{\prime}\right]^{+}$where $\mathrm{L}_{2}$ represents a chelating diphosphine ligand, was published. The detailed study included their synthesis from primary butatrienylidene precursors as well as their spectroscopic, electrochemical and structural properties [10]. From this study it was concluded that the iminium alkynyl resonance form III (Chart I) constitutes a major contributor to the bonding in these species. Evidence comes from (i) the shift of the IR valence band of the 


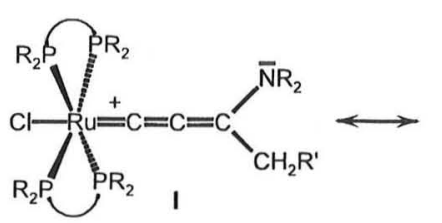

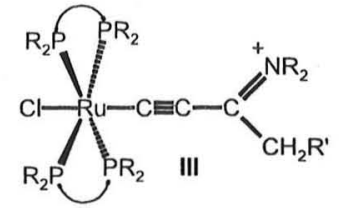

Chart I.

unsaturated carbon ligand to values rather close to those for the $\mathrm{C} \equiv \mathrm{C}$ stretch of $\mathrm{Ru}$ (II) alkynyl complexes, (ii) the observation of a $\mathrm{C}=\mathrm{N}$ band in the IR spectra, the assignment of which was supported by theoretical calculations applying density functional methods, (iii) an essentially planar coordination around the iminium nitrogen atom as established by X-ray crystallography, and (iv) the observation of a sizable energy barrier to rotation around the $\mathrm{C}=\mathrm{N}$ bond by proton and carbon NMR spectroscopies. However, their electronic transitions and excited states were not spectroscopically studied. In view of the efficient luminescence of other metal-alkynyl complexes described above, we thought it interesting to study the photophysical properties of such ruthenium(II)-aminoallenylidene complexes.

Here we present a combined resonance Raman study of the electronic transitions of two ruthenium(II)allenylidene derivatives and an investigation of the luminescence properties of a larger series of such complexes. The structures of the compounds that were employed are depicted in Chart II. Of particular interest is the observation of long-lived luminescence from higher-lying excited states of these complexes.

\section{Results and discussion}

Irrespective of the exact nature of the $\mathrm{ER}_{n}$ moiety $\left(\mathrm{ER}_{n}=\mathrm{NR}_{2}, \mathrm{SR}, \mathrm{SeR}\right.$, aryl, alkyl) [10-12], all of the allenylidene complexes trans $-\left[\mathrm{Cl}\left(\mathrm{L}_{2}\right)_{2} \mathrm{RuC}_{3}\left(\mathrm{ER}_{n}\right) \mathrm{R}^{\prime}\right]^{+}$ display two absorption bands of grossly different intensities in their optical spectra. As a representative example, the absorption spectrum of compound $\mathbf{3 b}$ in a MeOH:EtOH (4:1) mixture is shown in Fig. 1 in both fluid solution and as a solid glass. These bands have been assigned as the HOMO $\rightarrow$ LUMO and the HOMO-1 $\rightarrow$ LUMO excitations and the different absorptivities traced to the forbidden nature of the lowest energy transition as contrasted to the allowed excitation from the lower lying HOMO-1 level. According to quantum mechanical calculations both these transitions are associated with a sizable amount of charge transfer from the metal donor to the unsaturated organic acceptor ligand. Thus, the occupied frontier orbitals are mainly based on the $\left\{\mathrm{ClRu}\left(\mathrm{L}_{2}\right)_{2}\right\}$ entity with major contributions from the chloride and the ruthenium atom while the LUMO is delocalized over the entire allenylidene ligand with strong participation of the carbon atoms $\mathrm{C}^{1}, \mathrm{C}^{3}$ and the $\mathrm{ER}_{n}$ substituent [10]. Some of the amino substituted allenylidene complexes display another weaker absorption band superimposed on the low energy side of the intense HOMO-1 $\rightarrow$ LUMO transition. This feature has been assigned as the corresponding spin-forbidden singlet $\rightarrow$ triplet excitation belonging to the HOMO-1 $\rightarrow$ LUMO transition, again aided by time-dependent (TD) DFT calculations [10].

Chart II depicts the set of complexes that have been selected for this study. They have been chosen such as to cover a broad range of substitution patterns including congeners with only alkyl substituents on the nitrogen atom and the aliphatic side chain (1a,b), the (anthracenylmethyl)methylamine derivative (1c), the iminostilbene and 2,5-dimethylpyrrole derived complexes (2a,b) where the nitrogen atom is part of an unsaturated aromatic heterocycle, and some complexes with unsaturated non-aromatic or aromatic substituents in the side chain attached to the terminal carbon atom of the allenylidene ligand $(\mathbf{3 a}-\mathbf{c})$. Complexes $\mathbf{3} \mathbf{a}, \mathbf{b}$ comprise a tautomeric couple that is related by the migration of a hydrogen atom from the position at the ring junction to the exocyclic methylene group adjacent to the oxygen atom. This proton migration is an acid catalyzed, irreversible process with the aromatization of the unsaturated heterocycle as the driving force [13]. As a point of comparison the sulfur substituted allenylidene complex 4 [11] has also been included in this study.

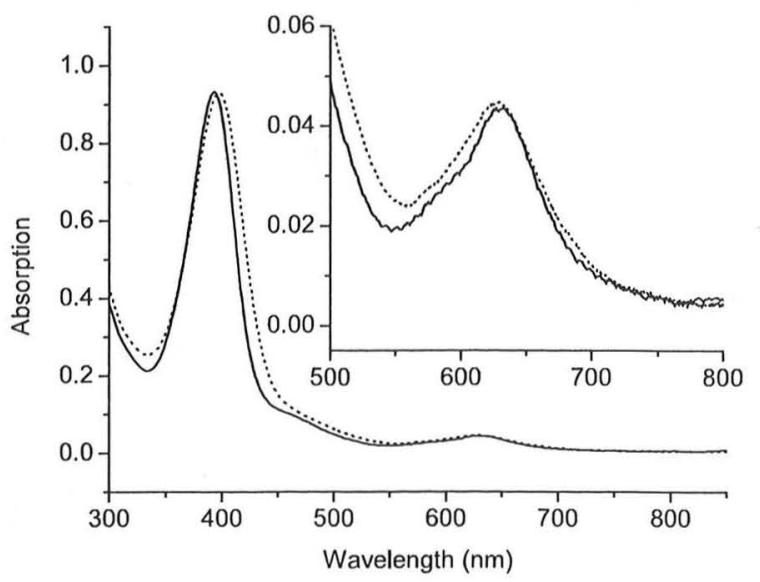

Fig. 1. Absorption spectrum of 3b in EtOH:MeOH (4:1) at $298 \mathrm{~K}$ (dotted) $93 \mathrm{~K}$ (drawn). The insert is an enlargement of the $500-800 \mathrm{~nm}$ region of the spectrum, showing the absorption band due to the $\mathrm{HOMO} \rightarrow$ LUMO transition. 

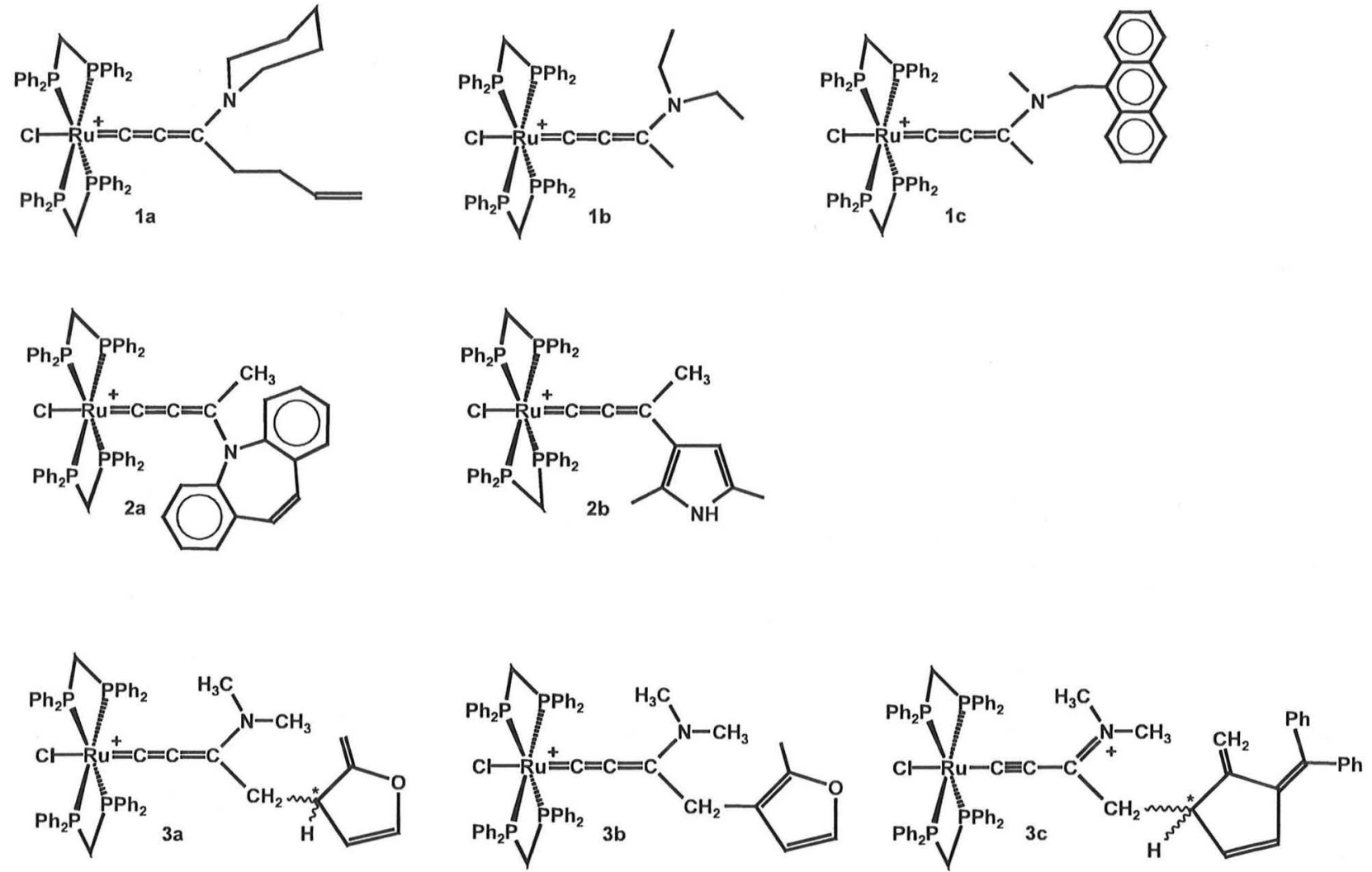

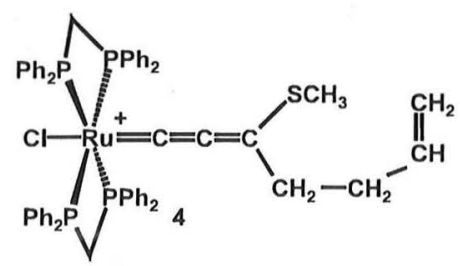

Chart II.

The main absorption band in the visible regime of the electronic spectra is centered at $390-408 \mathrm{~nm}$ for complexes $\mathbf{1 a}-\mathbf{c}$ and $\mathbf{3 a}-\mathbf{c}$, but shifted to significantly lower energies (432 and $522 \mathrm{~nm}$, respectively) for the iminostilbene [14] and the 2,5-dimethylpyrrole derived complexes $2 \mathbf{2 a}, \mathbf{b}$. The latter derivative may be viewed as a vinylogous aminoallenylidene complex.

The extension of its choromophore by an additional (intracyclic) double bond gives rise to a strong red shift of especially the HOMO-1 $\rightarrow$ LUMO band as compared to the other congeners within this series. In 1c the HOMO-1 $\rightarrow$ LUMO band is overlapped by the (anthracenylmethyl)amine based absorptions that present five sharp and distinct maxima at $\lambda=388,368,351,335$ and $320 \mathrm{~nm}$ spaced by some $1400-1450 \mathrm{~cm}^{-1}$ each. Vibrational progressions of this type are commonly observed for anthracene based chromophores. The slight red shift as compared to the parent amine agrees well with the lower electron density on the nitrogen atom upon its incorporation into complex 1c and the acquisition of some iminium type character. Similar effects have been noted upon protonation [15].

Virtually the only direct experimental evidence for the character of the electronic transition belonging to a certain absorption band comes from resonance Raman spectroscopy. The merit of this technique is based on the fact that in the resonance Raman spectrum, obtained by irradiation into a certain absorption band, only those vibrations that are influenced by the corresponding electronic transition are observed [16]. The availability of irradiation energies in our laboratory precluded the recording of resonance Raman spectra of the major part of the aminoallenylidene complexes since their absorption bands are located too far into the blue part of the spectrum. Hence, this part of our studies was limited to the pyrrole derivative $\mathbf{2} \mathbf{b}$ and the sulfur substituted 


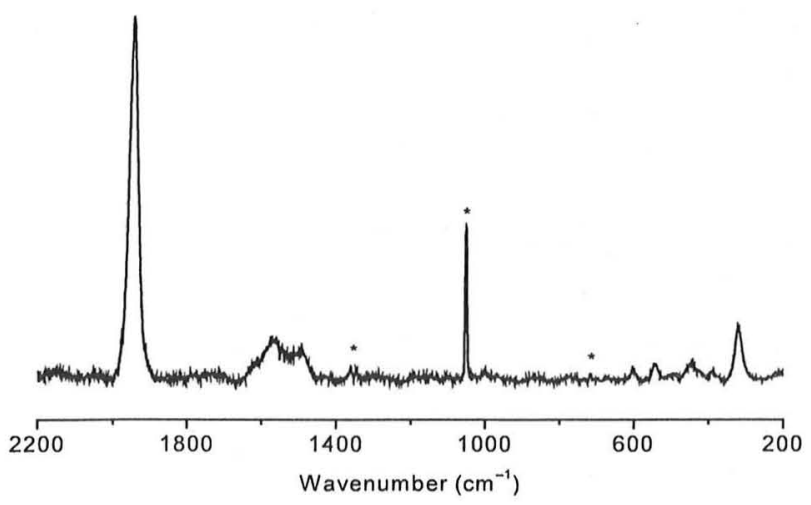

Fig. 2. Resonance Raman spectrum of 4 in $\mathrm{KNO}_{3}$, obtained by irradiation at $\lambda_{\mathrm{exc}}=477 \mathrm{~nm}$. Asterisks denote nitrate bands.

complex 4. The resonance Raman spectrum, obtained by irradiation into the $457 \mathrm{~nm}$ absorption band of 4 dispersed in $\mathrm{KNO}_{3}$, shows only a few resonantly enhanced bands (Fig. 2). The observation of a strong band due to the symmetric CCC stretching vibration at $1947 \mathrm{~cm}^{-1}$ clearly shows the involvement of the allenylidene moiety in the electronic transition. Very interestingly, a band is observed at $319 \mathrm{~cm}^{-1}$, which can be attributed to $v(\mathrm{Ru}-\mathrm{Cl})$. Its observation shows that the $\mathrm{Ru}-\mathrm{Cl}$ bond is affected by the electronic transition in agreement with the pronounced mixed metal-halide character of the HOMO-1 as found from DFT calculations [10]. Finally, a number of weaker bands are visible, of which the one at $597 \mathrm{~cm}^{-1}$ may be due to the $\mathrm{C}-\mathrm{S}$ stretch. None of the characteristic absorption bands of the phenyl substituents at the dppm ligands (dppm $=\mathrm{Ph}_{2} \mathrm{PCH}_{2} \mathrm{PPh}_{2}$ ) were detected. This agrees well with the quantum mechanical results which indicate only a small contribution from the phosphines to the overall electron density in any frontier orbital. For $\mathbf{2 b}$ several vibrational bands that arise from the pyrrole substituents were observed in addition to the CCC stretch at $1966 \mathrm{~cm}^{-1}$ and the $\mathrm{Ru}-\mathrm{Cl}$ stretch at 303 $\mathrm{cm}^{-1}$. Such bands are located at 1517 and $1362 \mathrm{~cm}^{-1}$ ( $v_{\text {ring }}$, out-of-phase and in-phase $\mathrm{C}=\mathrm{C}$ stretch), at 1062 $\mathrm{cm}^{-1}$ (in-plane $\mathrm{CH}$-deformation) and $1010-940 \mathrm{~cm}^{-1}$ (strong broad band, $\mathrm{CH}$-deformation and $\gamma_{\mathrm{CH}}$ ) and at 431,403 and $371 \mathrm{~cm}^{-1}(\gamma$ and $\delta$ modes of the pyrrole ring) [17]. This observation indicates that the pyrrole ring constitutes an integral part of the chromophore. In contrast to 4 , additional weak bands that can be traced to the dppm ligands at 1482 and $1431 \mathrm{~cm}^{-1}$ are also observed.

These resonance Raman results prove that the allowed HOMO-1 $\rightarrow$ LUMO transition has pronounced MLCT character. However, contrary to the platinum(II) and rhenium(I)-alkynyl complexes mentioned in the introduction, the compounds under study proved to be non- or only very weakly luminescent in fluid solution at room temperature. We therefore resorted to low tem- perature solvent glass measurements, under which conditions efficient thermally activated excited state decay paths should be eliminated. Upon cooling solutions of the complexes in 2-methyltetrahydrofuran (MTHF) to $77 \mathrm{~K}$ to a glassy state, the absorption bands due to the HOMO-1 $\rightarrow$ LUMO transitions sharpen but remain at essentially the same position as in the solution spectra. In contrast, a notable blue shift of the energies of the HOMO $\rightarrow$ LUMO absorptions by about 1900-2100 $\mathrm{cm}^{-1}$ compared to their solution spectra was observed in MTHF, while no such effect was observed in alcohol solvents. As an example the absorption spectrum of $\mathbf{3 b}$ in EtOH:MeOH (4:1) is given in Fig. 1, both in fluid solution and in low temperature glasss. Table 1 summarizes absorption data for the complexes in a rigid MTHF glass that was employed in most of the studies.

Steady-state emission spectra recorded on a glassy solution of $\mathbf{3 b}$ in EtOH:MeOH (4:1) show that depending on the excitation wavelength two different emission bands are observed (Fig. 3). On excitation of the HOMO $\rightarrow$ LUMO transition at around $600 \mathrm{~nm}$, an emission band in the near-infrared is observed at around $800 \mathrm{~nm}$. In contrast, irradiation at around $400 \mathrm{~nm}$ into the absorption band that belongs to the much stronger allowed HOMO-1 $\rightarrow$ LUMO transition, surprisingly, no emission is observed at this wavelength. Instead, a strong emission is observed at around $588 \mathrm{~nm}$. Excitation spectra, recorded on observing the $588 \mathrm{~nm}$ emission wavelength follow the absorption spectra quite closely. This proves that the emission is indeed due to $\mathbf{3 b}$ and

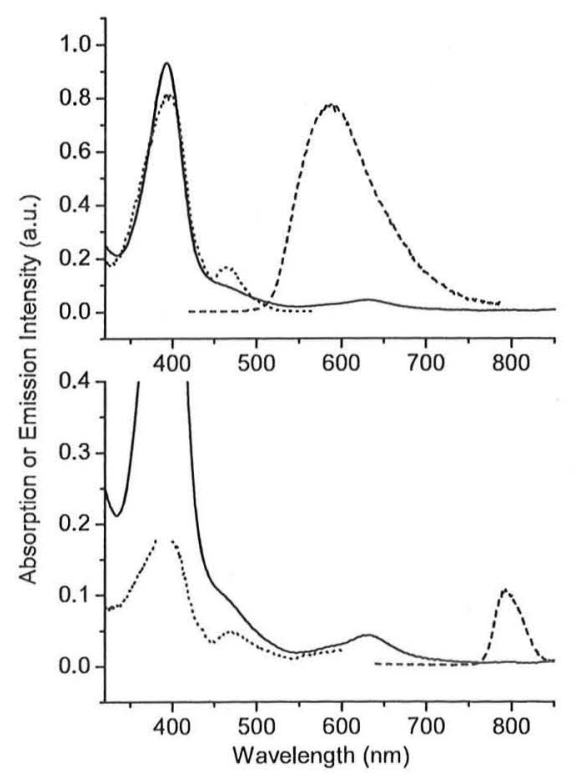

Fig. 3. Absorption (solid), emission (dashed), and excitation (dotted) spectra of 3b in EtOH:MeOH (4:1) at $93 \mathrm{~K}$. (Top) Emission spectrum: $\lambda_{\text {exc }}=400 \mathrm{~nm}$, excitation spectrum $\lambda_{\mathrm{em}}=580 \mathrm{~nm}$. (Bottom) Emission spectrum: $\lambda_{\mathrm{exc}}=630 \mathrm{~nm}$, excitation spectrum $\lambda_{\mathrm{em}}=780 \mathrm{~nm}$ (data near $\lambda=400 \mathrm{~nm}$ or $\lambda>600 \mathrm{~nm}$ could not be measured due to experimental restrictions, see text). 
Table 1

Absorption and emission data for allenylidene complexes in MeTHF at $77 \mathrm{~K}$

\begin{tabular}{|c|c|c|c|c|c|}
\hline Compound & $\lambda_{\mathrm{exc}}(\mathrm{nm})$ & $\lambda_{\max }(\mathrm{nm})^{\mathrm{a}}$ & $\lambda_{\mathrm{em}}(\mathrm{nm})$ & Apparent Stokes shift $\left(10^{3} \mathrm{~cm}^{-1}\right)$ & $\tau(\mu \mathrm{s})$ \\
\hline $1 \mathrm{a}$ & 454 & $392,460,582$ & 567 & 7.9 & 95 \\
\hline 1b & 454 & $394,453,595$ & 569 & 7.8 & 58 \\
\hline 1c & 419,477 & $388,477,557^{\mathrm{b}}$ & 614 & 9.5 & 0.72 \\
\hline $2 \mathrm{a}$ & 454 & $432,480,552$ & 618 & 7.0 & 46 \\
\hline $2 \mathbf{b}$ & 454 & $521,656,717^{\mathrm{b}}$ & None & & \\
\hline $3 a$ & 355 or $450^{\mathrm{c}}$ & $408,-, 631^{b}$ & 561 & 6.7 & 175 \\
\hline \multirow[t]{2}{*}{$3 \mathbf{b}$} & 405 or 450 & $393,453,642$ & 562 & 7.7 & 170 \\
\hline & 590 or 642 & & 810 & 3.2 & 65 \\
\hline \multirow[t]{2}{*}{$3 b^{d}$} & 400 & $393,466,632$ & 588 & 8.4 & n.d. ${ }^{\mathrm{e}}$ \\
\hline & 630 & & 794 & 3.2 & n.d. ${ }^{\mathrm{e}}$ \\
\hline $3 c$ & 355 or $450^{c}$ & $405,-, 478$ & 577 & 7.4 & 105 \\
\hline \multirow[t]{2}{*}{4} & 490 & $457,-, 582$ & 724 & 8.1 & 15 \\
\hline & 600 & & 807 & 4.8 & 1.3 \\
\hline
\end{tabular}

a $\mathrm{HOMO}-1 \rightarrow$ LUMO, singlet-triplet HOMO-1 $\rightarrow$ LUMO, HOMO $\rightarrow$ LUMO.

b In $\mathrm{CH}_{2} \mathrm{Cl}_{2}$ solution.

c No emission observed upon excitation at $590 \mathrm{~nm}$.

d In EtOH:MeOH (4:1) at $93 \mathrm{~K}$.

c Not determined.

cannot belong to any possible trace impurity present in the sample. Interestingly, in the excitation spectrum, the relative intensity of the band ascribed to the singlettriplet HOMO-1 $\rightarrow$ LUMO transition is enhanced with respect to the singlet-singlet transition when compared to the absorption spectrum. This means that the relative luminescence efficiency is higher upon excitation of the singlet-triplet transition, which can be an indication that inter-system crossing (ISC) to the emitting state is less than unity. Alternative processes from the optically populated singlet state include ISC to a different triplet state or nonradiative decay to the ground state. Monitoring the $800 \mathrm{~nm}$ emission band, the excitation spectrum follows again the absorption spectrum. There are several experimental problems in this case. First of all, the $400 \mathrm{~nm}$ absorption band region cannot be fully scanned, due to the use of grating optics in the luminescence spectrometer. Furthermore, the rhodamine quantum counter that is used as a reference in the excitation spectra is not usable above $600 \mathrm{~nm}$, prohibiting the recording of excitation spectra further toward the red. However, it is clear that both absorption bands contribute to the long-wavelength emission, to some extent.

On irradiation into the lowest-energy absorption band, which belongs to the HOMO $\rightarrow$ LUMO transition, luminescence was only observed in two cases, that of the furylmethyl derivative $\mathbf{3 b}$ and the sulfur substituted one 4. Indeed, judging from the low absorptivities of the absorption bands which are due to the symmetry forbidden transitions to the lowest singlet excited state, the radiative decay rate from the corresponding triplet state is expected to be very low, leading to a negligible luminescence quantum yield [18]. It is therefore all the more surprising that in the two mentioned cases luminescence is observed, which especially for $3 \mathbf{b}$ has quite a long lifetime $(\tau=65 \mu \mathrm{s})$. One reason for these long excited state lifetimes might be the limited distortion of the complexes in their excited states, which results in small vibrational overlap between ground and excited states and therefore slow nonradiative decay [19-22]. Indeed the apparent Stokes shift, i.e. the energy difference between absorption and emission, shows that the complexes $\mathbf{3 b}$ and $\mathbf{4}$ are only moderately distorted in their lowest triplet excited states.

As noticed above, exciting the complexes into their overlap allowed HOMO-1 $\rightarrow$ LUMO transition, surprisingly led to luminescence not from the lowest excited state but from a higher lying excited state, which is attributed to the second ${ }^{3}$ MLCT state. Excitation of the spin allowed singlet-singlet and the spin-forbidden singlet-triplet $\mathrm{HOMO}-1 \rightarrow$ LUMO transitions of a certain complex resulted in excited state lifetimes and qualitative quantum yields that were exactly the same, proving that intersystem crossing is very efficient. However, apparently internal conversion or intersystem crossing to the lowest triplet state is far from efficient, allowing the observation of luminescence from higherlying triplet excited states. One reason might be the relatively large energy gap between the two exicted states which slows the non-radiative transition process from one to the other. Although Kasha's rule states that luminescence should occur from the lowest state of a given multiplicity, in several other cases luminescence from higher excited states has been observed, e.g. for the complexes $\left[\mathrm{W}(\mathrm{CO})_{4}(\mathrm{NN})\right]$, where $\mathrm{NN}$ is 1,10 phenanthroline or 3,4,7,8-tetramethyl-1,10-phenanthroline [23], and also in several aryl-platinum(II) $\alpha$-diimine complexes [24]. The lifetimes are for the most part exceptionally long for ${ }^{3} \mathrm{MLCT}$ states, with values up to $175 \mu \mathrm{s}$ 
for complex 3a (Table 1). In comparison, the excited state lifetime of the prototypical MLCT complex $\left[\mathrm{Ru}(\mathrm{bpy})_{3}\right]^{2+}$ under similar conditions is only $5.3 \mu \mathrm{s}$ at similar emission energies [25]. Excited states with lifetimes of this magnitude are generally found for complexes that have ${ }^{3}$ MLCT states with significant admixtures of ${ }^{3} \mathrm{IL}$ (intraligand) states, such as $[\mathrm{Ru}(\mathrm{b}-$ py) ${ }_{2}$ (bpy-C $\equiv \mathrm{C}$-pyrene $\left.)\right]^{2+}(\tau=46 \mu$ s at room temperature) [26], or $\left[\mathrm{Ru}\left(\mathrm{CN}_{2}-\mathrm{np}\right)(\mathrm{bpy})_{2}\right]^{2+} \quad\left(\mathrm{CN}_{2}-\mathrm{np}=\right.$ naphtho[2,3- $f][1, \omega]$ phenanthroline-9,14-dicarbonitrile) ( $\tau=464 \mu$ s at $77 \mathrm{~K}$ ) [27]. The longevity of the emissions may arise from a favorable combination of two effects. On one hand all of the crucial frontier orbitals are delocalized over the whole $\mathrm{RuC}_{3}\left(\mathrm{ER}_{n}\right) \mathrm{R}^{\prime}\left(\mathrm{ER}_{n}=\mathrm{NR}_{2}\right.$, $\mathrm{SR}, \mathrm{SeR}$, alkyl, aryl) entity despite a distinct concentration of electron density on the $\left\{\mathrm{ClRu}\left(\mathrm{L}_{2}\right)\right\}$ moiety in the occupied and on the organic ligand in the LUMO levels. Secondly, charge transfer from the metal to the organic ligand is expected to cause only small structural changes within the $\mathrm{RuC}_{3}\left(\mathrm{ER}_{n}\right) \mathrm{R}^{\prime}$ entity. Optical charge transfer partly resembles the metal-centered one-electron oxidation which has been studied by electrochemical and spectroelectrochemical methods including the EPR spectroscopic identification of some of the radical dications. DFT calculations on the $18 \mathrm{VE}$ monocations and the open-shell $17 \mathrm{VE}$ dications have further revealed that the main structural changes upon one-electron oxidation are a shortening of the $\mathrm{Ru}-\mathrm{Cl}$ and the lengthening of the $\mathrm{Ru}-\mathrm{P}$ bonds while the $\mathrm{Ru}-\mathrm{C}, \mathrm{C}-\mathrm{C}$ and the $\mathrm{C}-\mathrm{N}$ bond parameters are left almost unchanged [10]. This delocalised nature of the frontier orbitals may result in the distortion of many bonds in the excited state, compared to the ground state. However, the distortion along any normal coordinate will be small, limiting vibrational overlap and thus slowing down non-radiative decay [20-22].

\section{Conclusions}

These studies have shown the remarkable occurrence of long-lived emission from higher-lying excited states of several heteroatom substituted ruthenium(II)-allenylidene complexes. In spite of this scientifically interesting finding, the required experimental conditions (low temperature glasses) and the moderate luminescence efficiencies, preclude the application of these complexes as luminophores.

\section{Experimental}

\subsection{Instrumentation}

Infrared spectra were obtained on a Perkin Elmer Paragon 1000 PC FT-IR instrument. ${ }^{1} \mathrm{H}-(250.13 \mathrm{MHz})$ and ${ }^{13} \mathrm{C}-(62.90 \mathrm{MHz})$ NMR spectra were recorded on a Bruker AC 250 spectrometer at $303 \mathrm{~K}$. The spectra were referenced to the residual protonated solvent $\left({ }^{1} \mathrm{H}\right)$ or the solvent signal itself $\left({ }^{13} \mathrm{C}\right)$. For complexes 1c, $2 \mathbf{a}$ [14] and $3 \mathbf{a}-\mathbf{c}$, the assignment of ${ }^{13} \mathrm{C}-\mathrm{NMR}$ spectra was aided by a DEPT-135 measurement. UV-vis experiments were performed on an Omega 10 spectrometer by Bruins Instruments in HELMA quartz cuvettes whith $1 \mathrm{~cm}$ optical path lengths. Elemental analysis $(\mathrm{C}, \mathrm{H}, \mathrm{N})$ was performed at in-house facilities. Resonance Raman spectra of the complexes dispersed in $\mathrm{KNO}_{3}$ pellets were recorded on a Dilor XY spectrometer equipped with a Wright Instruments CCD detector, using a Spectra Physics 2040E Ar ${ }^{+}$laser in combination with Coherent CR490 and CR590 dye lasers (with Coumarin 6 and Rhodamine $6 \mathrm{G}$ dyes) as excitation sources under a $180^{\circ}$ backscattering geometry. The pellet was spun in order to minimize thermal and photochemical decomposition. Data acquisition was controlled by Dilor Labspec 2.08 software. The spectra were calibrated using the Raman bands due to the symmetrical stretching and in plane bending vibrations of $\mathrm{NO}_{3}^{-}$(at 1051 and $716 \mathrm{~cm}^{-1}$, respectively) [28] and corrected for baseline deviations using Grams software. Continuouswave $(\mathrm{CW})$ emission spectra were obtained on a Spex 1681 fluorimeter, equipped with an Xe arc light source, a Hamamatsu R928 photomultiplier tube detector and double excitation and emission monochromators. Emission spectra were corrected for source intensity and detector response by standard correction curves. Excitation spectra were referenced to an internal rhodamine solution. Nanosecond time-resolved emission spectra were obtained using a setup described previously [29]. As irradiation sources the third harmonic $(355 \mathrm{~nm})$ of a Spectra Physics GCR3 Nd:YAG laser or a continuously tunable (400-700 nm) coherent infinity XPO laser were used. Solutions of the complexes in 2-methyltetrahydrofuran (MTHF) were directly immersed in liquid nitrogen in a quartz dewar.

\subsection{Procedures}

The synthesis of the new allenylidene complexes closely follows our established protocol [10]. As a representative, the procedure leading to compound $\mathbf{2 b}$ is described. cis $-\mathrm{RuCl}_{2}(\mathrm{dppm})_{2}$ [30] $(0.175 \mathrm{~g}, \quad 0.186$ mmol) and $\mathrm{NaSbF}_{6}(0.192 \mathrm{~g}, 0.744 \mathrm{mmol})$ were suspended in $\mathrm{PhCl}(40 \mathrm{ml})$ and excess butadiyne [31] were added by pipette. The suspension was stirred under ambient conditions for $30 \mathrm{~min}$ whereupon a green color developed. 2,5-Dimethylpyrrole (76 $\mu \mathrm{l}, 0.071 \mathrm{~g}, 0.744$ $\mathrm{mmol}$ ) were added by microsyringe and the suspension was left to stir under ambient conditions for 5 days during which time a gradual color change to deep purple was observed. The progress of the reaction was monitored by the developing intense IR band at $1973 \mathrm{~cm}^{-1}$ 
and stopped when subsequent measurements revealed no further intensity increase. The suspension was filtered and excess $\mathrm{NaSbF}_{6}$ and $\mathrm{NaCl}$ removed. The solvent was distilled off in vacuo and the oily residue taken up in $\mathrm{CH}_{2} \mathrm{Cl}_{2}$ and precipitated by the addition of $n$-hexane (60 $\mathrm{ml})$. The fine, deep purple powder was washed with copious amounts of ether. Two reprecipitations from $\mathrm{CH}_{2} \mathrm{Cl}_{2}$ by addition of ether to the rapidly stirred solutions gave $0.187 \mathrm{~g}(0.145 \mathrm{mmol}, 78 \%)$ of the analytically pure product. Data for $2 \mathrm{a}:{ }^{1} \mathrm{H}-\mathrm{NMR}(250$ $\mathrm{MHz}, \mathrm{CD}_{2} \mathrm{Cl}_{2}$ ): 1.23 (s, 3H, $\mathrm{CH}_{3}$ ), 1.97, 2.13 (each s, $3 \mathrm{H}$, $\mathrm{CH}_{3}$ (pyrrole)), 5.05 (dquint, $2 \mathrm{H}, J_{\mathrm{PC}}=4.2 \mathrm{~Hz}, J_{\mathrm{HH}}=$ $15.3 \mathrm{~Hz}, \mathrm{CH}_{2}(\mathrm{dppm})$ ), 5.21 (dquint, $2 \mathrm{H}, J_{\mathrm{PC}}=4.7 \mathrm{~Hz}$, $\left.J_{\mathrm{HH}}=15.3 \mathrm{~Hz}, \mathrm{CH}_{2}(\mathrm{dppm})\right), 7.20(\mathrm{~m}, 16 \mathrm{H}$, aryl-H (dppm)), 7.42-7.28 (m, $25 \mathrm{H}$, aryl-H (dppm) and 3-H (pyrrole)), 9.30 (br, 1H, NH); ${ }^{13} \mathrm{C}-\mathrm{NMR}(62.896 \mathrm{MHz}$, $\mathrm{CD}_{2} \mathrm{Cl}_{2}$ ): 12.0, 15.9 (each s, $\mathrm{CH}_{3}$ (pyrrole)), 30.0 (s, $\mathrm{CH}_{3}$ ), 47.9 (quint, $\left.J_{\mathrm{PC}}=11.4 \mathrm{~Hz}, \mathrm{CH}_{2}(\mathrm{dppm})\right), 109.6$ (s, $\mathrm{CH}$ (pyrrole)), 128.4 (vquint, $J_{\mathrm{PC}}=2.5 \mathrm{~Hz}, \mathrm{C}_{\text {meta }}(\mathrm{Ph}$, dppm)), 128.9 (vquint, $J_{\mathrm{PC}}=2.1 \mathrm{~Hz}, \mathrm{C}_{\text {meta }}(\mathrm{Ph}, \mathrm{dppm})$ ), 130.1 (s, $C\left(\mathrm{CH}_{3}\right)$, pyrrole), 130.4, 130.6 (each s, $\mathrm{C}_{\text {para }}$ $(\mathrm{Ph}, \mathrm{dppm})$ ), 132.0 (vquint, $J_{\mathrm{PC}}=12.0 \mathrm{~Hz}, \mathrm{C}_{\text {ipso }}(\mathrm{Ph}$, $\mathrm{dppm})$ ), 132.9 (vquint, $J_{\mathrm{PC}}=3.1 \mathrm{~Hz}, \mathrm{C}_{\text {ortho }}(\mathrm{Ph}, \mathrm{dppm})$ ), $133.1\left(\mathrm{~m}, \mathrm{C}_{\text {ipso }}(\mathrm{Ph}, \mathrm{dppm})\right), 133.3$ (vquint, $J_{\mathrm{PC}}=2.9 \mathrm{~Hz}$, $\left.\mathrm{C}_{\text {ortho }}(\mathrm{Ph}, \mathrm{dppm})\right), 142.5\left(\mathrm{~s}, C\left(\mathrm{CH}_{3}\right)\right.$, pyrrole $), 159.2$, 161.3 (each br, $\mathrm{Ru}=\mathrm{C}=C=\mathrm{C}$ and $\mathrm{Ru}=\mathrm{C}=\mathrm{C}=C$ ), 256.5 (quint, $\left.J_{\mathrm{PC}}=14.0 \mathrm{~Hz}, \mathrm{Ru}=C=\mathrm{C}=\mathrm{C}\right) ;{ }^{31} \mathrm{P}-\mathrm{NMR}(101.27$ $\left.\mathrm{MHz}, \mathrm{CD}_{2} \mathrm{Cl}_{2}\right):-8.7$ (s). IR $\left(\mathrm{KBr}, \mathrm{cm}^{-1}\right): 3371(\mathrm{~m}$, $\left.v_{\mathrm{NH}}\right), 1962$ (vs, $\left.v_{\mathrm{CCC}}\right), 1516\left(\mathrm{~s}, v_{\mathrm{C}=\mathrm{C}}\right.$, pyrrole $), 1473,1434$ (s, $v_{\mathrm{C}=\mathrm{C}}$, aryl), 1357 (s, $\left.v_{\mathrm{CC}, \text { pyrrole }}\right), 1096,988$ (s, $\mathrm{CH}_{\text {aryl, pyrrole }}$ ), 657 and 289 (vs, $\mathrm{SbF}_{6}^{-}$). UV-vis $\lambda_{\max }$ $\left(\mathrm{nm}, \varepsilon\left(\mathrm{M}^{-1} \mathrm{~cm}^{-1}\right), \mathrm{CH}_{2} \mathrm{Cl}_{2}\right): 656$ (1300), 521 (14500), 344 (7800), 271 (18500), 237 (22000). Anal. Calc. for $\mathrm{C}_{60} \mathrm{H}_{55} \mathrm{~F}_{6} \mathrm{ClNP}_{4} \mathrm{RuSb}: \mathrm{C}, 56.03 ; \mathrm{H}, 4.31 ; \mathrm{N}, 1.09$. Found: C, 55.57; H, 4.19; N, 1.02\%.

\section{Acknowledgements}

This work was supported by VW-Stiftung within their programme on 'Intra- and Intermolecular Electron Transfer'. R. F. W. thanks Professor Derk J. Stufkens for his kind hospitality during a stay at the Universiteit van Amsterdam.

\section{References}

[1] K.M.C. Wong, C.K. Hui, K.L. Yu, V.W.-W. Yam, Coord. Chem. Rev. 229 (2002) 123.
[2] M. Hissler, J.E. McGarrah, W.B. Connick, D.K. Geiger, S.D Cummings, R. Eisenberg, Coord. Chem. Rev. 208 (2000) 115.

[3] A. Klein, J. van Slageren, S. Záliš, Inorg. Chem. 41 (2002) 5216.

[4] J. van Slageren, A. Klein, S. Záliš, Coord. Chem. Rev. 230 (2002) 193.

[5] C.E. Whittle, J.A. Weinstein, M.W. George, K.S. Schanze, Inorg Chem. 27 (2001) 4053.

[6] V.W.-W. Yam, Chem. Commun. (2001) 789.

[7] T.E. Müller, S.W.-K. Choi, D.M.P. Mingos, D. Murphy, D.J. Williams, V.W.-W. Yam, J. Organomet. Chem. 484 (1994) 209.

[8] M.J. Irwin, J.J. Vittal, R.J. Puddephatt, Organometallics 16 (1997) 3541.

[9] V.W.-W. Yam, Acc. Chem. Res. 35 (2002) 555 (and references therein).

[10] R.F. Winter, K.-W. Klinkhammer, S. Záliš, Organometallics 20 (2001) 1317.

[11] R.F. Winter, Eur. J. Inorg. Chem. (1999) 2121.

[12] S. Hartmann, R.F. Winter, T. Scheiring, M. Wanner, J. Organomet. Chem. 637-639 (2001) 240.

[13] R. Harbort, S. Hartmann, R.F. Winter, K.-W. Klinkhammer, manuscript in preparation.

[14] R.F. Winter, S. Hartmann, S. Záliš, K.-W. Klinkhammer, Dalton Trans., submitted for publication

[15] S. Quici, A. Manfredi, M. Maestri, I. Manet, P. Passaniti, V. Balzani, Eur. J. Org. Chem. (2000) 2041.

[16] R.J.H. Clark, T.J. Dines, Angew. Chem. Int. Ed. Engl. 25 (1986) 131.

[17] D. Lin-Vien, N.B. Colthup, W.G. Fateley, J.G. Grasselli, The Handbook of Infrared and Raman Characteristic Frequencies of Organic Molecules, Academic Press, San Diego, 1991.

[18] S.J. Strickler, R.A. Berg, J. Chem. Phys. 37 (1962) 814

[19] J. van Slageren, D.J. Stufkens, Inorg. Chem. 40 (2001) 277.

[20] N.H. Damrauer, T.R. Boussie, M. Devenney, J.K. McCusker, J. Am. Chem. Soc. 119 (1997) 8253.

[21] J.A. Treadway, B. Loeb, R. Lopez, P.A. Anderson, F.R. Keene, T.J. Meyer, Inorg. Chem. 35 (1996) 2242.

[22] G.F. Strouse, J.R. Schoonover, R. Duesing, S. Boyde, W.E. Jones, Jr, T.J. Meyer, Inorg. Chem. 34 (1995) 473.

[23] I.R. Farrell, J. van Slageren, S. Záliš, A. Vlček, Jr, Inorg. Chim. Acta 315 (2001) 44.

[24] J. van Slageren, A. Klein, Eur. J. Inorg. Chem., submitted for publication.

[25] R.H. Fabian, D.M. Klassen, R.W. Sonntag, Inorg. Chem. 19 (1980) 1977.

[26] A. Harriman, M. Hissler, A. Khatyr, R. Ziessel, Chem. Commun. (1999) 735.

[27] G. Albano, P. Belser, L. De Cola, M.T. Gandolfi, J. Chem. Soc. Chem. Commun. (1999) 1171

[28] R. Frech, J.P. Devlin, Chem. Phys. Lett. 38 (1976) 79.

[29] C.J. Kleverlaan, D.J. Stufkens, I.P. Clark, M.W. George, J.J. Turner, D.M. Martino, H. van Willigen, A. Vlček, Jr, J. Am. Chem. Soc. 120 (1998) 10871.

[30] B. Chaudret, G. Commenges, R. Poilblanc, J. Chem. Soc. Dalton Trans. (1984) 1635.

[31] K.K. Georgieff, Y. Richard, Can. J. Chem. 36 (1958) 1280 . 